\title{
Post-flight test results of acousto-optic modulator devices subjected to Space Exposure
}

\author{
Narasimha S. Prasad ${ }^{* 1}$, Sudhir Trivedi ${ }^{2}$, Jolanta Rosemeier ${ }^{2}$, and Mark Diestler ${ }^{3}$ \\ ${ }^{1}$ NASA Langley Research Center, 5 N. Dryden St., MS 468, Hampton VA, 23681 \\ ${ }^{2}$ Brimrose Technology Corporation, 19 Loveton Circle, Sparks, MD 21152
}

\begin{abstract}
The objective of the Materials International Space Station Experiment (MISSE) is to study the performance of novel materials when subjected to the synergistic effects of the harsh space environment for several months. MISSE missions provide an opportunity for developing space qualifiable materials. Several laser and lidar components were sent by NASA Langley Research Center (LaRC) as a part of the MISSE 7 mission. The MISSE 7 module was transported to the international space station (ISS) via STS 129 mission that was launched on Nov 16, 2009. Later, the MISSE 7 module was brought back to the earth via the STS 134 that landed on June 1, 2011. The MISSE 7 module that was subjected to exposure in a space environment for more than one and a half years included fiber laser, solid-state laser gain materials, detectors, and semiconductor laser diode. Performance testing of these components is now progressing. In this paper, the results of performance testing of a laser diode module sent by NASA Langley Research Center on MISSE 7 mission will be discussed. This paper will present the comparison of pre-flight and post-flight performance of two different COTS acousto-optic modulator (AOM) devices. Post-flight measurements indicate that these two devices did not undergo any significant performance degradation.
\end{abstract}

Keywords: MISSE 7, Space Qualification, Lidar components, International Space Station (ISS), STS-129, STS-134

\section{INTRODUCTION}

The goal of the MISSE program is to evaluate the performance, stability, and long-term survivability of materials and components planned for use by NASA, the Department of Defense (DOD), other federal agencies and private entities on future Low Earth Orbit (LEO), synchronous orbit, and interplanetary space missions. The study of combined effects of radiation, ultraviolet (UV) light and atomic oxygen in space due to long term exposures will help in developing space qualifiable elements for future space missions. In-situ space testing is critical since it is difficult and not economical to conduct these studies inside simulated terrestrial facilities. The development of new generations of materials will allow our nation to continue to maintain technological superiority related to space endeavors.

\section{AN OVERVIEW OF THE MISSE PROGRAM}

MISSE is a series of experiments and so far, five MISSE missions have been successfully completed. More than 1500 samples have been tested on the MISSE project. Samples include chemicals, sensor devices, opto-mechanical elements, polymers, coatings, and biological materials and species, and composites. The MISSE project has also provided educational opportunities for students. MISSEs 3 and 4 have transported approximately 8 million basil seeds for science experiments of children to stimulate interest in space science.

A suitcase shaped rugged box known as a Passive Experiment Container (PEC) is used to transport the selected materials to and from the ISS. PECs were originally developed and used by NASA's Langley Research Center, Hampton, Virginia, for the ISS Phase I Risk Mitigation Program experiments conducted on the Russian Mir space station. Specific steps required for transporting materials include specimen preparation, fixing specimens inside a holder, integration of specimen holder on a tray, integration of trays in a PEC and integration of a PEC inside a carrier for ease of transport. All these tasks are carried out in a clean room environment.

\footnotetext{
*narasimha.s.prasad@nasa.gov; Phone 757-864-9403; Fax 757-864-8828.
} 
The PEC is tested for survivability under launch conditions by subjecting it to an appropriate shock and vibration environments. Subsequent to several months of space exposure, the PEC will be transported back to the Earth to undergo tests. The resulting test characteristics when compared with original characteristics before launch will provide an understanding of their survivability in space conditions. Furthermore, it will provide insights into required space qualification processes for future space applications.

During a space walk, also known as extra-vehicular activity (EVA) (i.e., when an astronaut works outside of a spacecraft), the PECs are attached to the handrails or at a specific location that is exposed to the space environment. Two possible mounting positions known as ram and wake are available. The materials in the ram side will be predominantly subjected to the atomic oxygen environment where as those materials in the wake side will mostly undergo UV exposure. After exposure in space, the MISSE PEC is retrieved in the same manner as it was deployed. The material samples are tested in laboratory conditions to see if they still possess their unique properties needed to complete space missions. MISSE PECs have active and passive detectors to give a time-history reading, or a reading of what happened to the materials at certain points in time. Back on the ground, tests will be conducted to determine the effects of its exposure for several months in space. The passive detectors report a cumulative measure of the following environments that the test specimens are exposed to namely UV exposure, atomic oxygen exposure, molecular contamination, tray temperature and man-made debris.

Terrestrial laboratories may provide limited environmental test conditions while in space, the components are simultaneously exposed to several conditions which normally are difficult to simulate. In many cases, the combined effect of being exposed to all of the environmental elements at once would provide insights into ruggedization for future space applications. The details of MISSE 1 to MISSE 6 missions can be found at NASA's MISSE webpage [2]. NASA's Langley Research Center (LaRC), Hampton, Virginia has managed the MISSE projects till recently. NASA LaRC successfully completed the MISSE 6 mission and supported follow-on MISSE 7 and 8 missions.

\section{SALIENT FEATURES OF THE MISSE 7 MISSION}

MISSE-7 is a suite of experiments that include over 700 new and affordable materials. The selected MISSE 7 specimens also have potential use in advanced systems for space applications. For MISSE 7 there are two PECs, 7A and 7B, which will be mounted on the outside of the ISS and hold samples on both sides of the PECs. PEC 7A's orientation will be zenith/nadir (space facing/Earth facing) while PEC 7B will face ram/wake (forward/backward) relative to the ISS orbit

Primary Responsibilities of MISSE 7A and 7B were carried out by Naval Research Laboratory and Air Force Research Laboratory, respectively. Glenn Research Center managed the MISSE 7 mission. NASA LaRC and Boeing played key roles. Besides the above agencies, industry collaborators, and academia had experiments on MISSE 7B. NASA LaRC provided a flight worthy package with laser and lidar components along with other logistics support. This effort at NASA LaRC was a follow-on effort to MISSE 6 mission [3].

Figure 1 shows the laser and lidar components that were sent on MISSE 7 missions. The flight worthy package includes a 1.5 micron Fiber Laser, a coherent receiver, several laser gain media, two acousto-optic modulators, an electro-optic modulator, and a high power laser diode bar. NASA LaRC is involved with Active Sensing of CO2 over Nights, Days (ASCENDS) and other lidar programs. ASCENDS is a mid-size, tier II future NASA mission [4]. Currently, several of these components are being used in ASCENDS related airborne and field test campaigns. These components have potential to be incorporated into future space based instrumentation dependent on their space qualification tests. 


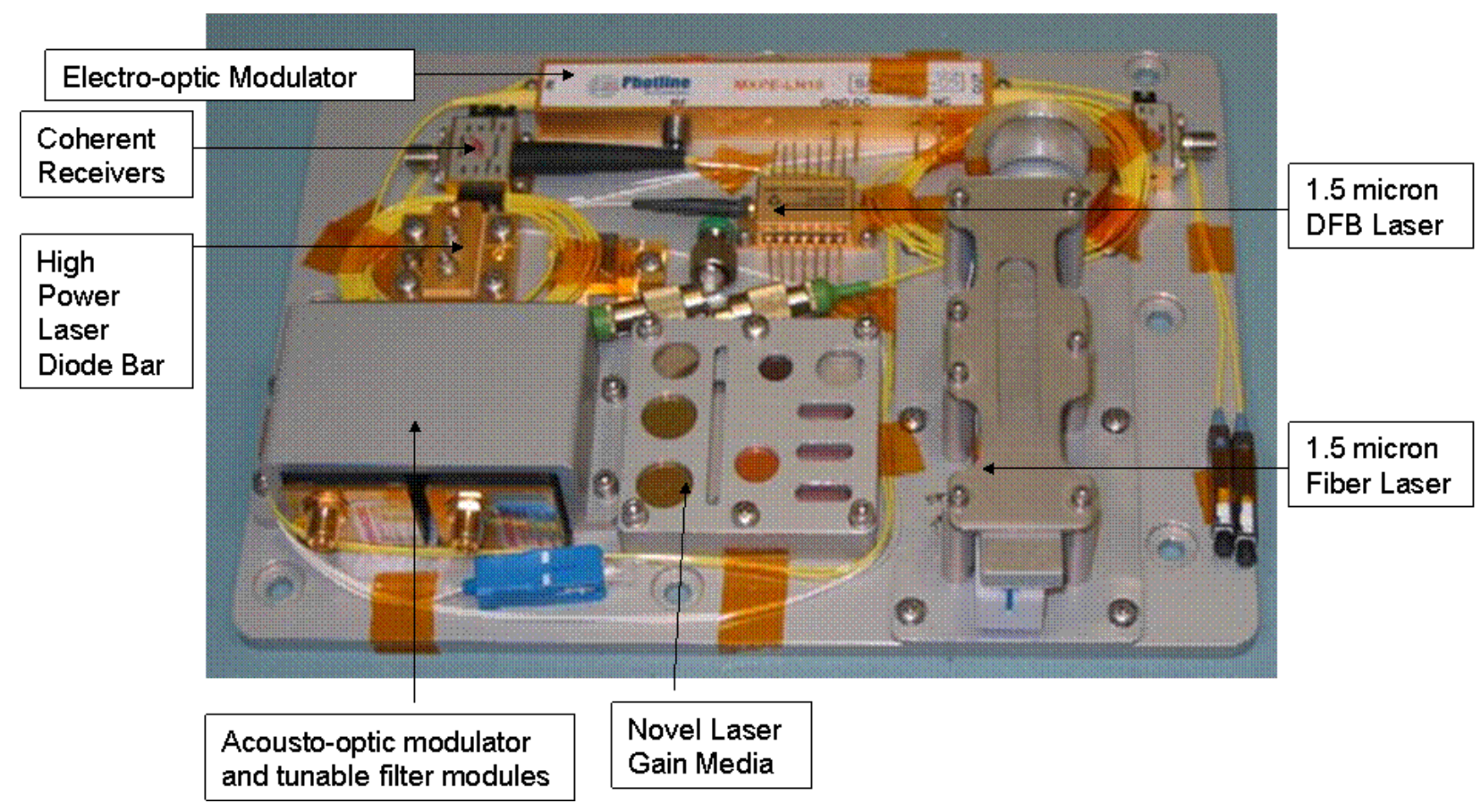

Figure 1. Laser and Lidar components mounted on a flight worthy package that was sent by NASA LaRC.

\section{MISSE 7 LAUNCH, RETURN AND RETRIEVAL}

MISSE-7 was mounted to the ISS's exterior on an EXPRESS Logistics Carrier (ELC). The power and data were provided by the ISS. It used the ISS communication system uplink/downlink capabilities to receive commands and downlink data. For this, no crew interaction was required. MISSE-7 was launched on Space Shuttle mission STS-129. MISSE 7A \& 7B were attached outside the ISS on the EXPRESS Logistics Carrier 2 (ECL2) on the S3 truss, and then opened and exposed to space during a spacewalk on November, 23, 2009. MISSE 7, consisting of PEC 7A and PEC 7B, was retrieved by spacewalking astronauts on STS 134 during the early morning hours of May 20, 2011 from their external operating location on the ISS and transferred to the STS-134 Shuttle payload bay. Further details on the shuttle missions related to MISSE missions can be found in references [5-8]. During its 1.5 year on-orbit mission, MISSE-7 has tested a variety of next-generation fiber lasers, solar cells and electronic devices and provided real-time downlink of science data. MISSE-7 has also continuously exposed cutting-edge material samples that will be analyzed in ground laboratories to determine how well they survived the space exposure effects of atomic oxygen, ultraviolet exposure,

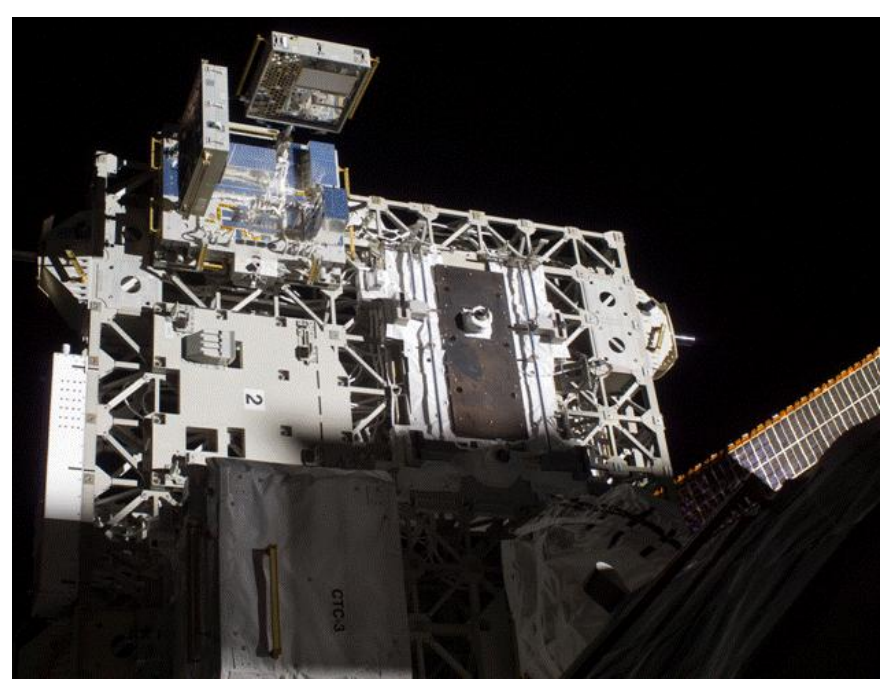
particle irradiation, and extreme temperature cycles. Figure 2 shows the on-orbit pictures of MISSE 7 taken by the space shuttle crew. The retrieved MISSE 7 package was disassembled at Marshall Space Flight Center. In November, 2011, the test unit from LaRC was returned for further testing. Post-flight testing of individual components on this package is now underway. Previously, post flight test results of the seed laser diode module and balanced detector were presented $[9,10]$. Details of measured environmental exposures can be found in reference [11]. In the following section, results of performance testing of two COTS acousto-optic modulators obtained from Brimrose Corporation of America [12] are discussed.

Figure 2. MISSE 7 on orbit. 


\section{ACOUSTO-OPTIC MODULATOR (AOM) DEVICES}

Acousto-optic (AO) devices are used in laser based optical systems for electronic control of the intensity, spectral characteristics and position of laser beams. Description of acousto-optic devices including acousto-optic modulators can be found in the literature $[13,14]$. When an acoustic wave is launched into an AO medium by an RF frequency generator via a piezoelectric transducer, refractive index variation that behaves like a sinusoidal grating is produced An incident laser beam passing through this grating will be diffracted into several orders (Raman-Nath several orders, and Bragg type - First order). Its angular position is linearly proportional to the acoustic frequency, so that the higher the frequency, the larger the diffracted angle. With appropriate design, the first order beam can offer the highest efficiency. The angle between the incident laser beam and the diffracted laser beam, $\Theta$, is given by $\Theta=\left[\left(\lambda \cdot f_{a}\right) / V_{a}\right]$, where $\lambda$, is the optical wavelength in air, $f_{a}$, is the acoustic frequency, and, $V_{a}$, is the acoustic velocity and the angle is measured with respect to the acoustic wave direction propagating at the base of the triangle formed by the three vectors. The amount of light diffracted by the acoustic wave depends on the intensity of the sound. Hence, the intensity of the acoustic wave can be used for laser beam modulation. The intensity of light diffracted (deflected) is proportional to the acoustic power $\left(\mathrm{P}_{\mathrm{ac}}\right)$, the material figure of merit $\left(\mathrm{M}_{2}\right)$, geometric factors $(\mathrm{L} / \mathrm{H})$ and inversely proportional to the square of the wavelength. For acousto-optic modulators (AOMs), the principal performance parameter is the modulation speed which is primarily determined by the transit time, $t$. The transit time, $t$, and the rise time, $\mathrm{t}_{\mathrm{r}}$, are given by: $\mathrm{t}-\mathrm{V} / \mathrm{d}$ and $\mathrm{t}_{\mathrm{r}}=0.85 \mathrm{t}$. To obtain a high modulation speed, $t$ should be as small as possible. The acousto-optic modulator can be used to shutter the laser beam "on" and "off" by an external digital TTL signal. Modulation Transfer Function is often used to study modulation characteristics. In the following sections, post-flight measurements of AOM devices are discussed.

\section{AOM MEASUREMENTS}

Two AOM devices were tested for diffraction efficiency and impedance characteristics. The diffraction efficiency was measured over the designed frequency span and impedance matching parameters using a network analyzer for assessing RF related losses. The AO materials of the two test devices were AMTIR-1 and $\mathrm{TeO}_{2}$. AMTIR-1 is a glass and is the acronym for amorphous material transmitting IR radiation. AMTIR-1, designated as AMF in our measurements is composed of Ge-As-Se with a transmission range of $0.7-12$ microns and acousto-optic figure of merit $(\mathrm{FOM}) \sim 164 \times 1 \mathrm{E}-15 \mathrm{~m}^{2} / \mathrm{W}$. The AO material properties of AMTIR-1 and $\mathrm{TeO}_{2}$, designated as TEF, can be found at [15]. The diffraction Efficiency, operational bandwidth, and impedance Matching properties were tested. Tests were for longitudinal mode operation. The diffraction efficiency is computed by measuring the intensity of light diffracted in a given order and the input power. It is proportional to the acoustic power $\left(\mathrm{P}_{\mathrm{ac}}\right)$, the material figure of merit $\left(\mathrm{M}_{2}\right)$, geometric factors $(\mathrm{L} / \mathrm{H})$ and inversely proportional to the square of the wavelength. The diffraction efficiency, $\eta$ is given by

$$
\eta=\sin ^{2}\left[\left(\frac{\pi}{\lambda}\right) \cdot\left(\frac{\mathrm{M}_{2} \cdot \mathrm{P}_{\mathrm{ac}} \cdot \mathrm{L}}{2 \cdot \mathrm{H}}\right)^{1 / 2}\right]
$$

Figure 3 shows the dimensions and two test devices. Table 1 shows the AOM test parameters.

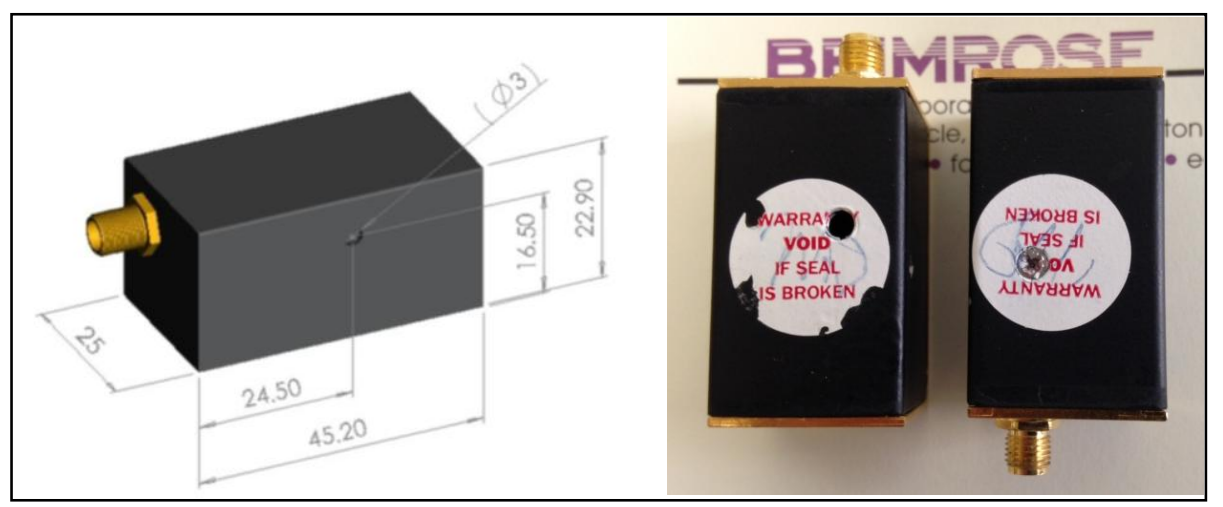

Figure 3. AOM device dimensions and devices that were returned after exposed in space environment. 
Table 1. AOM test parameters.

\begin{tabular}{|l|c|c|c|}
\hline \multicolumn{1}{|c|}{ Parameter } & AMF & TEF & Units \\
\hline Wavelength & 1523 & 488 & $\mathrm{~nm}$ \\
\hline Optical Power & 2.5 & 2.5 & $\mathrm{~mW}$ \\
\hline Beam Aperture & 0.5 & 1 & $\mathrm{~mm}$ \\
\hline RF Power & 0.5 & 1 & $\mathrm{~W}$ \\
\hline RF Freqency Sweep & $90-110$ & $180-280$ & $\mathrm{MHz}$ \\
\hline Detector & InGaAS & $\mathrm{Si}$ & \\
\hline & & & \\
\hline
\end{tabular}

Using a network analyzer, Smith chart and VSWR characteristics were measured. RF reflection losses that could arise from piezoelectric transducer element degradation due to space exposure can be evaluated from impedance matching measurements. The Smith Chart is a reflection coefficient diagram. Impedance is plotted on the Smith chart by normalizing to the characteristic impedance of the system which is $50 \mathrm{ohms}$. In a $50 \mathrm{ohm}$ system the center of the Smith chart is a pure 50 ohms. The characteristics tell us how close impedance matching our AOM devices is to $50 \Omega$. The design goal is to minimize RF reflections back to the transducer and maximize forward RF power to generate Acoustic waves inside the acousto-optic materials. Next, the VSWR is an acronym that stands for Voltage Standing Wave Ratio, is the ratio of the reflected voltage over the incident voltage. Lower the VSWR the better it is to reduce RF related losses. In the following discussions, both pre-flight and post-flight measurements of Diffraction efficiency, Smith chart, and VSWR are presented. Figure 4 show the setup used for diffraction efficiency and impedance matching measurements.

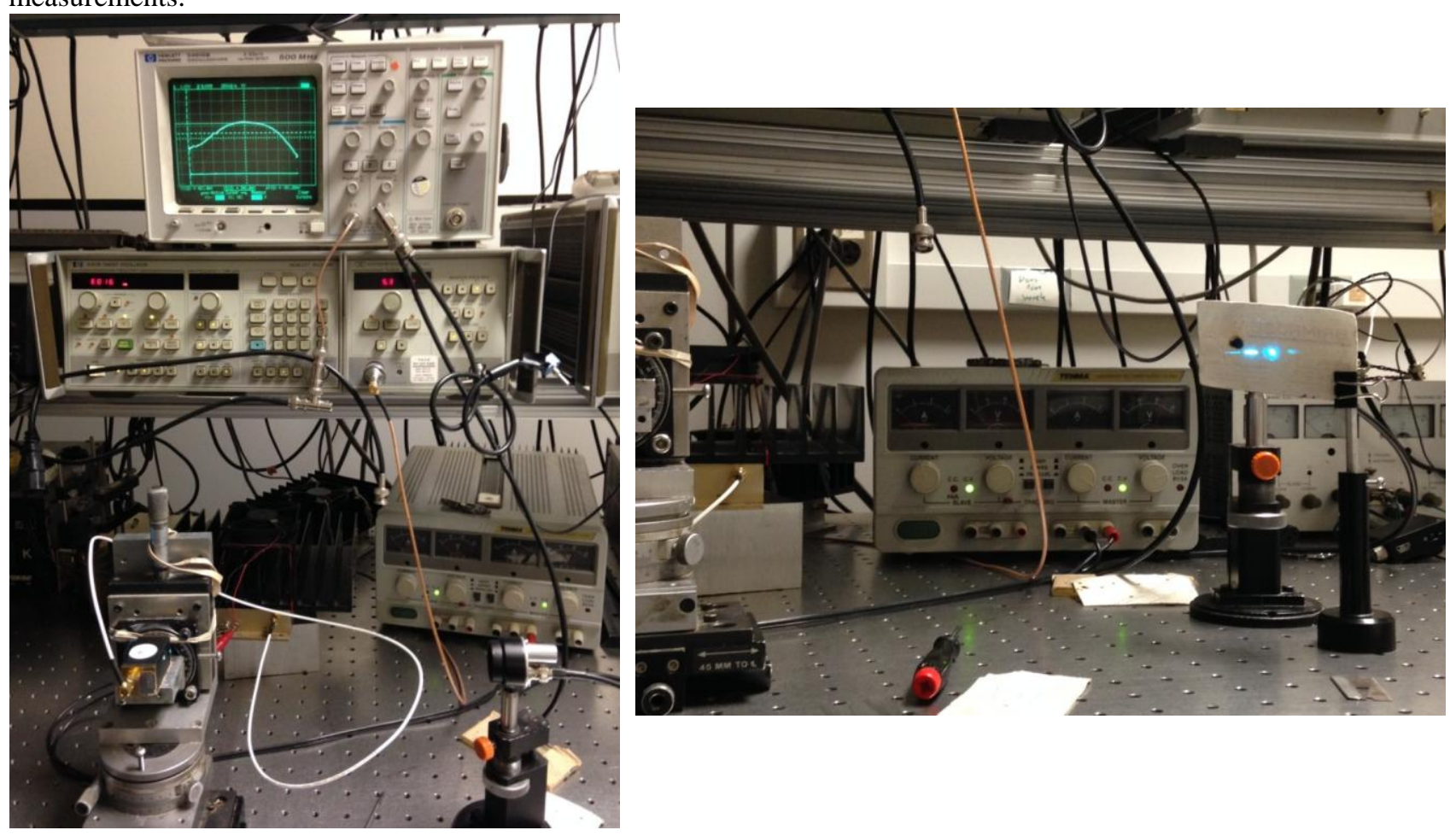

Figure 4. Test set-ups for measuring impedance matching (left) and diffraction efficiency (right) parameters. 
With the test parameters shown in Table 1, the following three sets of graphs are related to $\mathrm{TeO}_{2}$ (TEF). Figure 5 shows a comparison of Diffraction efficiency and bandwidth for the pre- and post-flight operations. Figures 6 and 7 show the Smith chart and VSWR measurements done at the room temperature. As one can see, no significant degradation is the device operation was noticed.
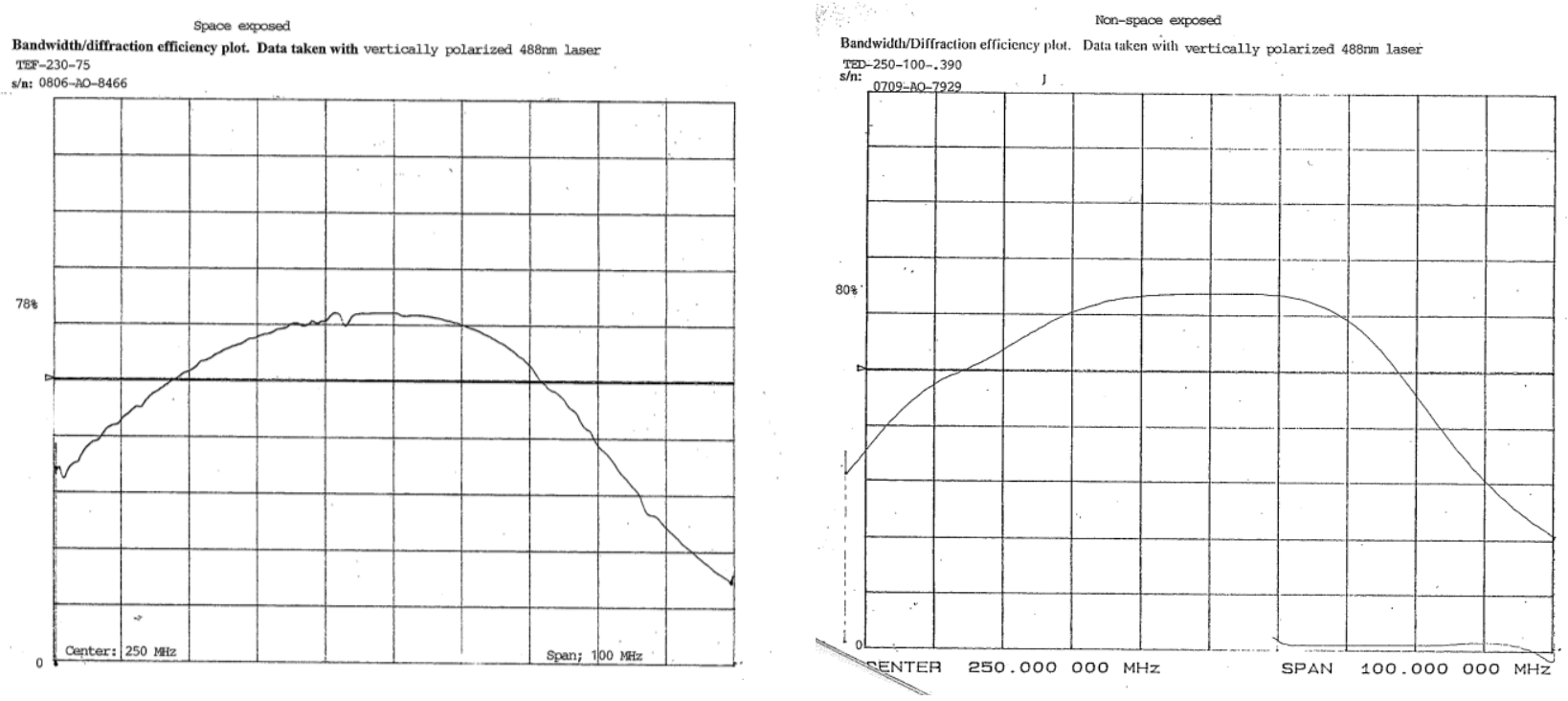

Figure 5. A comparison of Diffraction efficiency and operational bandwidth measured using a vertically polarized $488 \mathrm{~nm}$ for the post flight and pre-flight conditions.
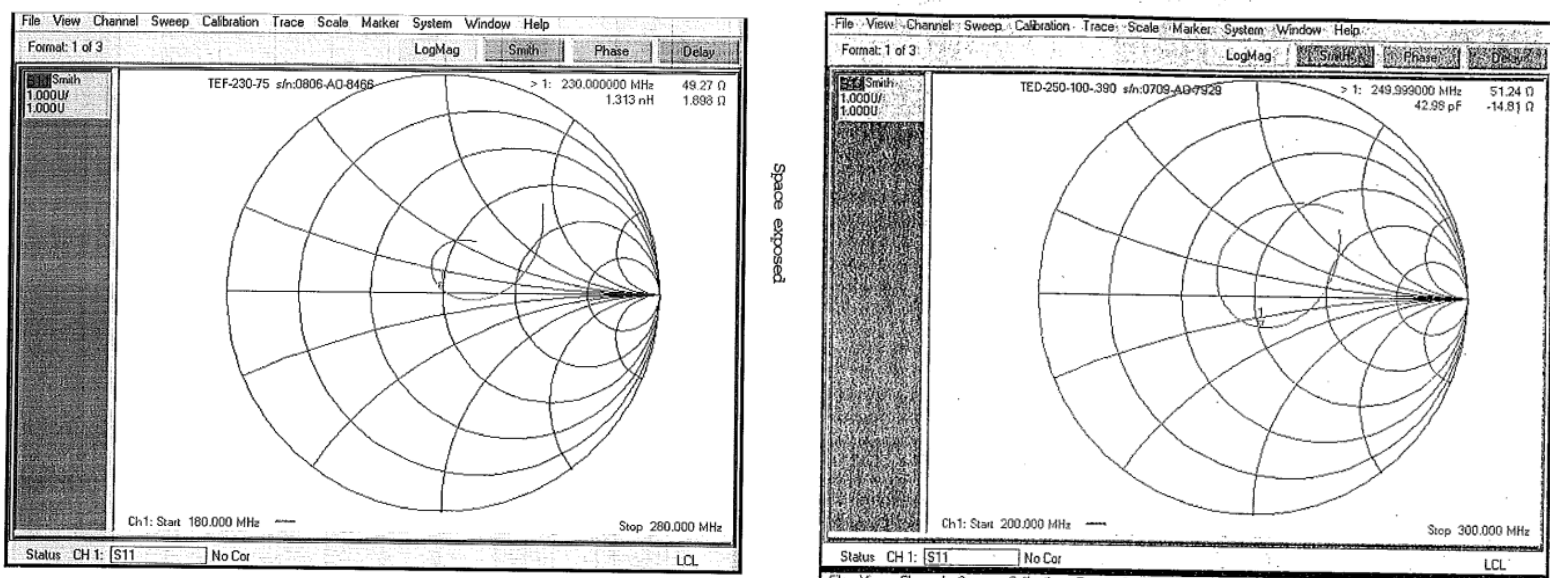

Figure 6. A comparison of Smith charts for the post and pre-flight operations. 

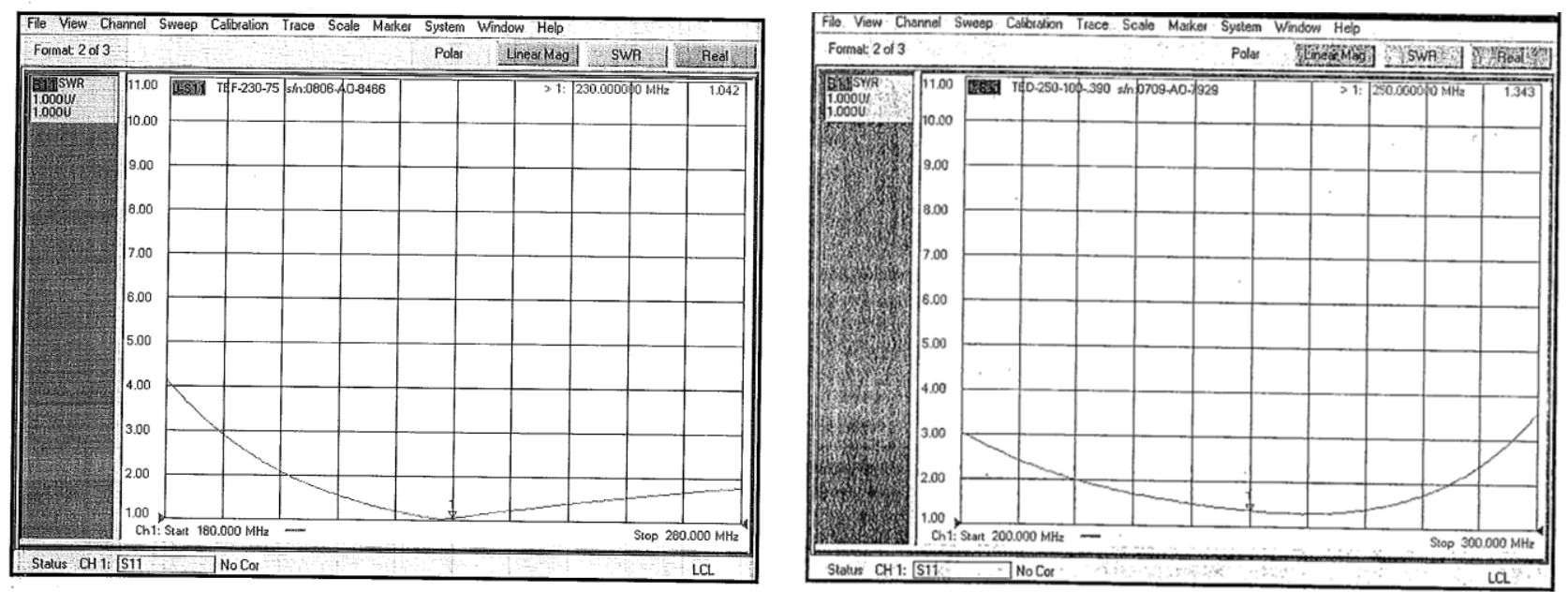

Figure 7. A comparison of VSWR measurements over $\sim 180 \mathrm{MHz}$ to $280 \mathrm{MHz}$ for post (left) and pre-flight (right) conditions. Lower the VSWR, the better it is to reduce RF related losses.

The following graphs are for the AMTIR-1 AOM device (AMF). Figure 8 is for the diffraction efficiency and operational bandwidth, Figure 9 and Figure 10 are the Smith chart and VSWR measurement for the AMTIR-1 AOM device. Even in this case, no significant degradation due to space exposure has been observed.
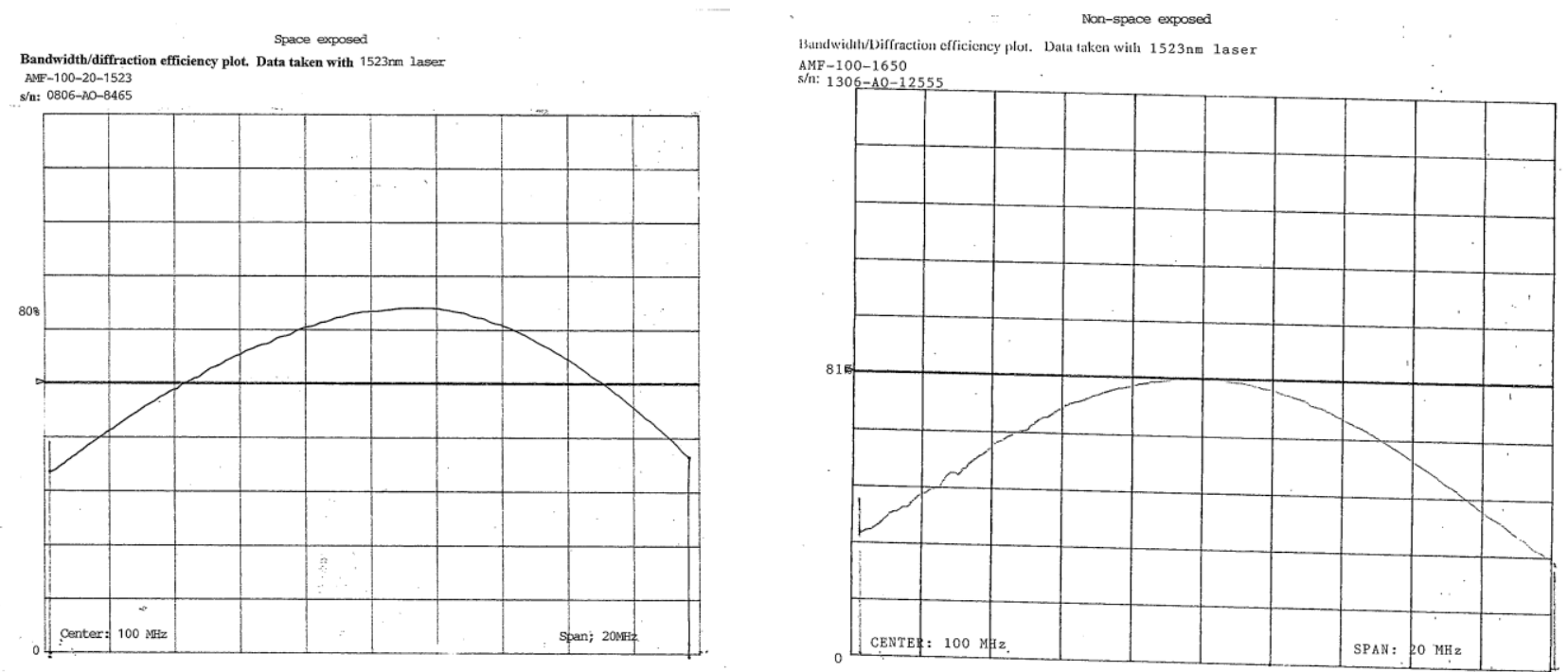

Figure 8. A comparison of diffraction efficiency and operational bandwidth characteristics for post (left) and pre-flight conditions. Data was taken at $1523 \mathrm{~nm}$ wavelength. 

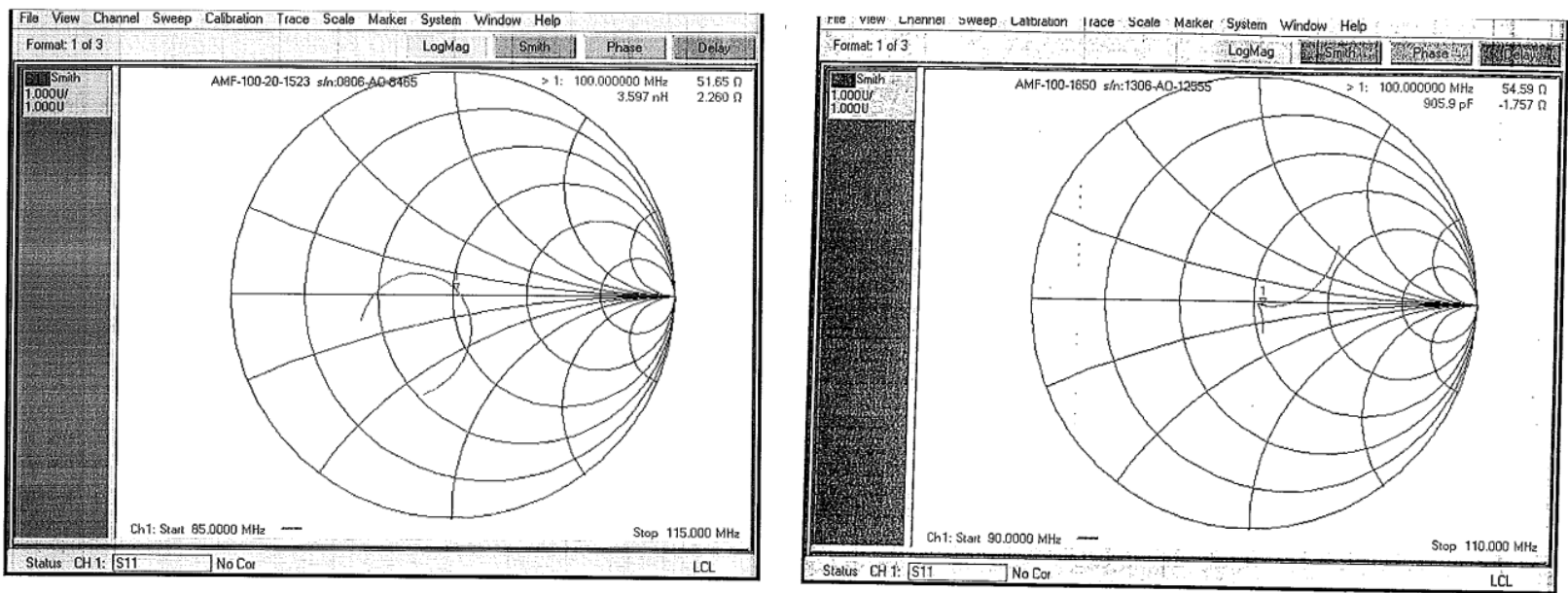

Figure 9. A comparison of Smith chart for post (left) and pre-flight (right) conditions of AMTIR-1.
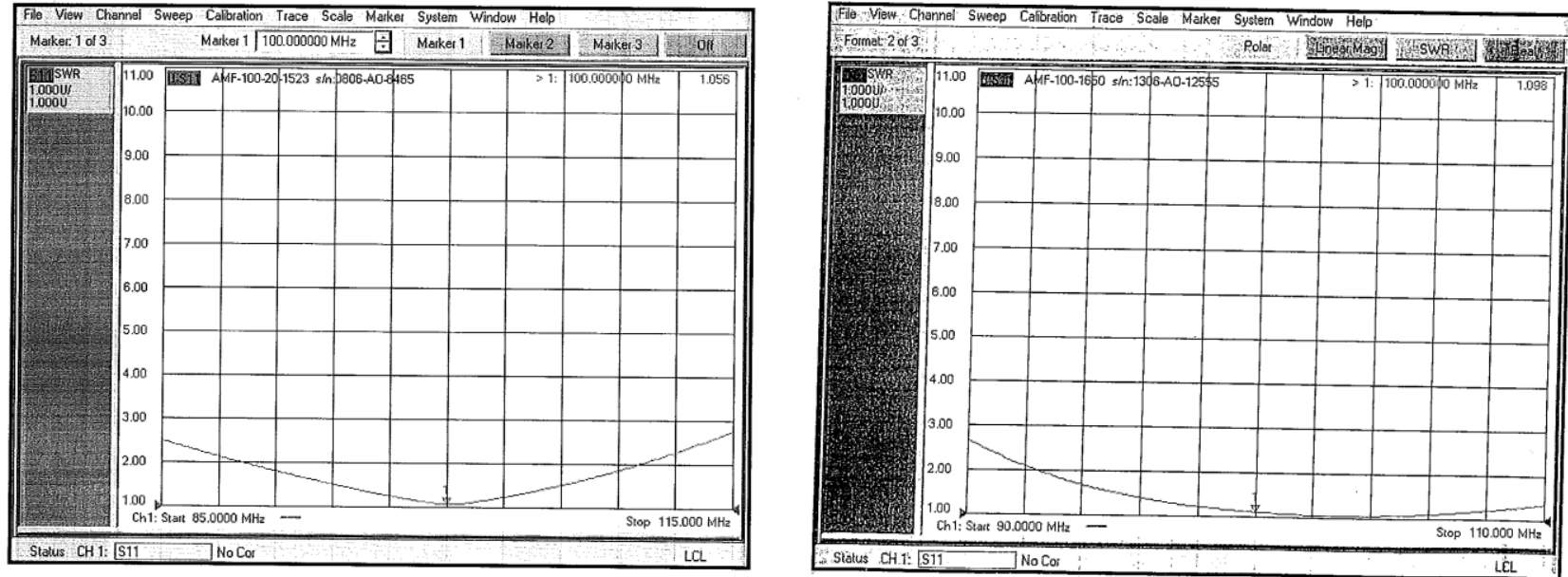

Figure 10. A comparison of VSWR measurements over $\sim 85 \mathrm{MHz}$ to $115 \mathrm{MHz}$ for post (left) and pre-flight (right) conditions. Lower the VSWR the better it is to reduce RF related losses.

\section{SUMMARY AND CONCLUSIONS}

The objective of the MISSE program is to study the performance, stability, and long-term survivability of novel materials when subjected to the synergistic effects of the harsh space environment. So far, seven MISSE missions have been completed. The MISSE 7 mission was launched in November 2009 and returned to the Earth in May 2011 on STS 134. Several specimens and devices for use in Laser and Lidar systems for potential use in ASCENDS and other future NASA missions were selected. These specimens will undergo similar tests to look into deviations from pre-exposure characteristics. In this paper, the post and pre-flight performances of two COTS AOM devices utilized in the MISSE 7 mission are discussed. The two AOMs devices were constructed using $\mathrm{TeO}_{2}$ and AMTIR-1 materials. Diffraction Efficiency, bandwidth, and impedance matching parameters were tested. No appreciable degradation due to space exposure were noticed. With suitable changes, these COTS devices can be considered for space operations. 


\section{ACKNOWLEDGMENTS}

The authors acknowledge William H. Kinard for providing guidance and support in the initial stages of Misse 7 mission development at NASA LaRC. Also, the authors thank Karen Gibson and Terry Clark for mechanical engineering support.

\section{REFERENCES}

[1] William H. Kinard, "MIR Environmental Effects Payload (MEEP) Archive System,” NASA, Langley Research Center, Hampton, Virginia. (http://setas-www.larc.nasa.gov/meep/meep.html and http://www.nasa.gov/centers/langley/news/factsheets/misse 2005.html).

[2] The NASA MISSE website: http://misseone.larc.nasa.gov/.

[3] Narasimha S. Prasad and William H. Kinard, "MISSE 6-Testing Materials in Space," Proc. SPIE 7095, 7095OD (2008).

[4] "Active Sensing of $\mathrm{CO}_{2}$ Emissions over Nights, Days, and Seasons (ASCENDS) Mission", NASA Science Definition and Planning Workshop, July 23-25, 2008, Univ. of Michigan, Ann Arbor, MI, available at http://decadal.gsfc.nasa.gov/documents/12-30-08-ASCENDS_Workshop.pdf

[5] STS-123 MCC Status Report \#15 (http://www.nasa.gov/mission_pages/shuttle/shuttlemissions/sts123/news/STS123-15.html).

[6] STS-123 MCC Status Report \#25 (http://www.nasa.gov/mission_pages/shuttle/shuttlemissions/sts123/news/STS123-25.html).

[7] http://www.nasa.gov/mission_pages/station/science/misse2009.html.

[8] http://www.nasa.gov/mission_pages/station/science/misse.html.

[9] Narasimha S. Prasad, "Performance testing of lidar components subjected to exposure in space via MISSE 7 mission," Proc. SPIE 8519, Nanophotonics and Macrophotonics for Space Environments VI, 851900 (October 15, 2012).

[10] Narasimha S. Prasad, "Post-flight test results of seed laser module subjected to space exposure," Proc. SPIE 8876, Nanophotonics and Macrophotonics for Space Environments VII, 88760C (24 September 2013).

[11] Finckenor, M. M., Moore, C., Norwood, J. K., Henrie, B. and de Groh, K., "Estimated Environmental Exposures for MISSE-7B," Proceedings of the 2012 National Space and Missile Materials Symposium (NSMMS) held June 2528, 2012 in Tampa, FL, August 2012. (Presentation format)

[12] For AOM data datasheets, please see e.g., http://www.brimrose.com/products/acousto_optics.html

[13] Amnon Yariv and Pochi Yeh, "Optical Waves in Crystals," John Wiley \& Sons, Inc., New York (1984).

${ }^{114]}$ B. E. A Saleh and M. C Teich, "Fundamentals of Photonics," John Wiley \& Sons, Inc., New York (1991).

[15] Handbook of Infrared Optical Materials, edited by Paul Klocek, CRC Press (1991). 